\title{
BOUNDS FOR RIEMANN-STIELTJES INTEGRALS
}

\author{
PAUL R. BEESACK
}

Abstract. Let $f, g, h$ be real valued functions on a compact interval $[a, b]$, where $h$ is of bounded variation with total variation $V$ on $[a, b]$, and such that $\int_{a}^{b} f d g$ and $\int_{a}^{b} h f d g$ both exist. If $m=\inf \{h(x): a \leqq x \leqq b\}$ it is shown that

$$
\begin{gathered}
\int_{a}^{b} h f d g \leqq m \int_{a}^{b} f d g+V \sup _{a \leqq \alpha<\beta \leqq b} \int_{\alpha}^{\beta} f d g, \\
\int_{a}^{b} h f d g \geqq m \int_{a}^{b} f d g+V \inf _{a \leqq \alpha<\beta \leqq b} \int_{\alpha}^{\beta} f d g .
\end{gathered}
$$

Corresponding bounds hold for improper Riemann-Stieltjes integrals. The first of the inequalities above extends a result of $R$. Darst and H. Pollard, who dealt with the case $f(x) \equiv 1$, and $g$ continuous on $[a, b]$.

In a recent paper [2], Darst and Pollard proved that if $h$ is real and of bounded variation on the interval $[a, b]$ and $g$ is continuous there, then

$$
\int_{a}^{b} h d g \leqq(\inf h)[g(b)-g(a)]+V(h ;[a, b]) S_{g}(a, b),
$$

where $V$ is the total variation of $h$ on $[a, b]$, and

$$
S_{g}(a, b)=\sup _{a \leqq \alpha<\beta \leqq b} \int_{\alpha}^{\beta} d g .
$$

Although it was not pointed out in [2], by replacing $g$ in (1) by $(-g)$, one also obtains

$$
\int_{a}^{b} h d g \geqq(i n f h)[g(b)-g(a)]+V(h ;[a, b]) s_{g}(a, b),
$$

where

$$
s_{g}(a, b)=\inf _{a \leqq \alpha<\beta \leqq b} \int_{\alpha}^{\beta} d g .
$$

Received by the editors January $11,1972$.

AMS (MOS) subject classifications (1970). Primary 26A42, 26A86; Secondary 26A45.

Key words and phrases. Riemann-Stieltjes integral, second integral mean value theorem. 
The inequalities ( 1$),\left(l^{\prime}\right)$ thus give upper and lower bounds for $\int_{a}^{b} h d g$, analogous to those given in the "second integral mean value theorem" of R. P. Boas [1, p. 4] . (See also Widder [4, p. 18] .)

It is the purpose of this note to show that the bounds $(1),\left(1^{\prime}\right)$ remain valid even if $g$ is not continuous on $[a, b]$, provided only that $g$ is bounded on $[a, b]$ and $\int_{a}^{b} h d g$ exists. A careful examination of the proof in [2] shows that the continuity of $g$ was only used at two points of the proof: first, to justify the assumption that $h(\xi)=0$ for some $\xi \in[a, b]$ in the second reduction step of the proof; finally, to justify the existence of the integral $\int_{a}^{b} h d \varphi$ (since the continuity of $\varphi$ follows from the continuity of $g$ ).

We observe first that when $g$ is bounded with $g(a)=0$, and if

$$
\varphi(t) \equiv \inf _{a \leqq \xi \leqq t} g(\xi), \quad a \leqq t \leqq b,
$$

it follows that $\varphi$ is monotone decreasing on $[a, b]$. Also one easily verifies that $\varphi$ is left-continuous, right-continuous, or continuous at each point $t \in[a, b]$ at which $g$ has the corresponding property. Now the existence of $\int_{a}^{b} h d g$ implies that $h$ and $g$ have no common points of left- or of right-discontinuity on $[a, b]$ if $\int_{a}^{b} h d g$ is defined in the Pollard-Moore sense as a limit under successive refinement of partitions, or that $h$ and $g$ have no common points of discontinuity if $\int_{a}^{b} h d g$ is defined as a limit taken as the norm of partitions tends to zero. It follows that $h$ and $\varphi$ also have no common points of discontinuity of the same character, and since $h$ and $\varphi$ are of bounded variation on $[a, b], \int_{a}^{b} h d \varphi$ exists. (See, for example Hildebrandt [3, pp. 50,56,66].) The continuity of $g$ is thus not essential for the final steps of the proof in [2].

In order to complete the proof of our assertion, it suffices to rearrange the proof in [2] somewhat in order to avoid the necessity of assuming that $h$ vanishes at some point of $[a, b]$ when $m=\inf h(x)$ $=0$. As in [2], the general case of (1) follows from the case $m=0$, so we are to prove that

$$
\int_{a}^{b} h d g \leqq V(h ;[a, b]) S_{g}(a, b)
$$

when $\inf h(x)=0$. Given any integer $n \geqq 1$ there exists $\xi_{n} \in[a, b]$ such that $\lim h\left(\xi_{n}\right)=0$. We now write

$$
\begin{aligned}
\int_{a}^{b} h d g=\int_{a}^{\xi_{n}} h d g+\int_{-b}^{-\xi_{n}} h_{1} d \mu, \\
\left(h_{1}(x) \equiv h(-x), \mu(x) \equiv-g(-x)\right),
\end{aligned}
$$


and note that $h$ and $h_{1}$ are nonnegative on their respective intervals of integration, and that $V\left(h_{1} ;\left[-b,-\xi_{n}\right]\right)=V\left(h ;\left[\xi_{n}, b\right]\right)$ and $S_{\mu}\left(-b,-\xi_{n}\right)=S_{g}\left(\xi_{n}, b\right)$, just as in [2]. Noting that we may assume that $g(a)=0$ since $\int_{a}^{b} h d g=\int_{a}^{b} h d(g-g(a))$, and defining $\varphi$ on $\left[a, \xi_{n}\right]$ as above, and $\psi(t) \equiv g(t)-\varphi(t)$, it follows as in [2] that

$$
\begin{aligned}
\int_{a}^{\xi_{n}} h d g & \leqq h\left(\xi_{n}\right) \psi\left(\xi_{n}\right)+S_{g}\left(a, \xi_{n}\right) V\left(h ;\left[a, \xi_{n}\right]\right) \\
& \leqq h\left(\xi_{n}\right) S_{g}\left(a, \xi_{n}\right)+S_{g}\left(a, \xi_{n}\right) V\left(h ;\left[a, \xi_{n}\right]\right)
\end{aligned}
$$

Proceeding in the same way on $\left[-b,-\xi_{n}\right]$, we similarly obtain

$$
\begin{aligned}
\int_{-b}^{-\xi_{n}} h_{1} d \mu \leqq & h_{1}\left(-\xi_{n}\right) S_{\mu}\left(-b,-\xi_{n}\right) \\
& +S_{\mu}\left(-b,-\xi_{n}\right) V\left(h_{1} ;\left[-b,-\xi_{n}\right]\right) \\
= & h\left(\xi_{n}\right) S_{g}\left(\xi_{n}, b\right)+S_{g}\left(\xi_{n}, b\right) V\left(h ;\left[\xi_{n}, b\right]\right) .
\end{aligned}
$$

It follows that for each $n \geqq 1$,

$$
\int_{a}^{b} h d g \leqq 2 h\left(\xi_{n}\right) S_{g}(a, b)+S_{g}(a, b) V(h ;[a, b]),
$$

so that (3) follows on letting $n \rightarrow \infty$.

Because of the usefulness of bounds of the form (1), (1'), it may be worthwhile pointing out the following extensions.

Corollary 1. Let $h$ be of bounded variation on $[a, b]$, and let $f$ and $g$ be any functions such that $\int_{a}^{b} f d g$ and $\int_{a}^{b} h f d g$ both exist. If $m=\inf \{h(x): a \leqq x \leqq b\}$, then

$$
\begin{aligned}
& \int_{a}^{b} h f d g \leqq m \int_{a}^{b} f d g+V(h ;[a, b]) \sup _{a \leqq \alpha<\beta \leqq b} \int_{\alpha}^{\beta} f d g, \\
& \int_{a}^{b} h f d g \geqq \int_{a}^{b} f d g+V(h ;[a, b]) \inf _{a \leqq \alpha<\beta \leqq b} \int_{\alpha}^{\beta} f d g .
\end{aligned}
$$

This follows from (1) and $\left(1^{\prime}\right)$ applied to $\int_{a}^{b} h d G$, where $G(x) \equiv$ $\int_{a}^{x} f d g$; note that by [3, p. 53] $\int_{a}^{b} h d G$ exists and equals $\int_{a}^{b} h f d g$.

This result may also be extended to improper integrals,

$$
\int_{a}^{b-} F d \mu \equiv \lim _{c \rightarrow b-} \int_{a}^{c} F d \mu
$$


where $-\infty<a<b \leqq+\infty$, and $\int_{a}^{c} F d \mu$ exists for each $c \in(a, b)$. We define $V(h ;[a, b)) \equiv \lim _{c \rightarrow b-} V(h ;[a, c])$, and say that $h$ is of bounded variation on $[a, b)$ if this limit is finite.

Conollary 2. Suppose that $h$ is of bounded variation on $[a, b)$, that $\int_{a}^{b-} f d g$ exists, and that $\int_{a}^{c} h f d g$ exists for each $c \in(a, b)$. If $G(x) \equiv \int_{a}^{x} f d g$ is bounded on $[a, b)$, then $\int_{a}^{b-} h f d g$ exists, and

$$
\begin{aligned}
& \int_{a}^{b-} h f d g \leqq m \int_{a}^{b-} f d g+V(h ;[a, b)) \sup _{a \leqq \alpha<\beta<b} \int_{\alpha}^{\beta} f d g, \\
& \int_{a}^{b-} h f d g \geqq m \int_{a}^{b-} f d g+V(h ;[a, b)) \inf _{a \leqq \alpha<\beta<b} \int_{\alpha}^{\beta} f d g,
\end{aligned}
$$

where $m=\inf \{h(x): a \leqq x<b\}$ is (necessarily) finite.

It is easy to see that $h$ is bounded on $[a, b)$, and even that $h(b-)$ exists and is finite. By writing

$$
\int_{a}^{c} h f d g=\int_{a}^{c} h d G=h(c) G(c)-\int_{a}^{c} G d h,
$$

we see that $\int_{a}^{b-} h f d g$ exists, the improper integral $\int_{a}^{b-} G d h$ being absolutely convergent. We now apply Corollary 1 on the interval $[a, c]$ for $a<c<b$ to obtain (for example) the upper bound

$$
\int_{a}^{c} h f d g \leqq m(c) \int_{a}^{c} f d g+V(h ;[a, c]) \sup _{a \leqq \alpha<\beta \leqq c} \int_{\alpha}^{\beta} f d g,
$$

where $m(c)=\inf \{h(x): a \leqq x \leqq c\}$. By considering the two cases $m=h(b-), m<h(b-)$, it can be shown that $\lim _{c \rightarrow b-} m(c)=m$, and the result now follows readily.

\section{REFERENCES}

1. R. P. Boas, Jr., The Jensen-Steffensen inequality, Univ. Beograd. Publ. Elektrotehn. Fak. Ser. Mat. Fiz. No. 302-319 (1970), 1-8. MR 45 \#2117.

2. R. Darst and H. Pollard, An inequality for the Riemann-Stieltjes integral, Proc. Amer. Math. Soc. 25 (1970), 912-913. MR 41 \#5565.

3. T. H. Hildebrandt, Introduction to the theory of integration, Pure and Appl. Math., vol. 13, Academic Press, New York, 1963. MR 27 \#4900.

4. D. V. Widder, The Laplace transform, Princeton Math. Series, vol. 6, Princeton Univ. Press, Princeton, N. J., 1941. MR 3, 232.

Carleton University, Ottawa 1 , Ontario, Canada 